Nathaniel W. Yang, MD

Department of Otorhinolaryngology

College of Medicine - Philippine General Hospital University of the Philippines Manila

Department of Otolaryngology - Head and Neck Surgery Far Eastern University - Nicanor Reyes Medical Foundation Institute of Medicine
Correspondence: Dr. Nathaniel W. Yang Department of Otorhinolaryngology Ward 10, Philippine General Hospital University of the Philippines Manila Taft Avenue, Ermita, Manila 1000

Philippines

Phone: (632) 85264360

Fax: (632) 85255444

Email: nathaniel.w.yang@gmail.com

The author declared that this represents original material, that the manuscript has been read and approved by the author, that the requirements for authorship have been met by the author, and that the author believes that the manuscript represents honest work.

Disclosures: The author signed a disclosure that there are no financial or other (including personal) relationships, intellectual passion, political or religious beliefs, and institutional affiliations that might lead to a conflict of interest.

\section{On the Importance of Proper Window and Level Settings in Temporal Bone CT Imaging}

During a discussion on temporal bone imaging, a group of resident trainees in otolaryngology were asked to corroborate the finding of a fracture in set of images that were supposed to be representative of a fracture involving the otic capsule.' (Figure 1)

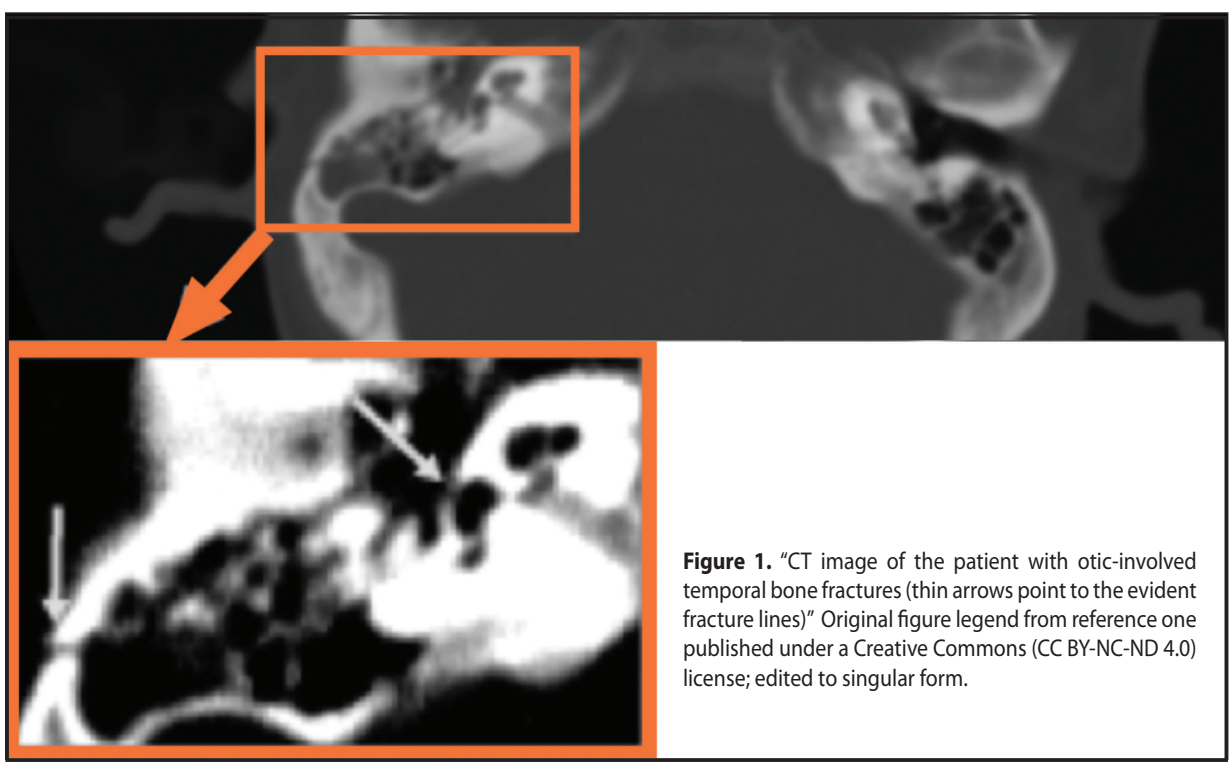

Their comments included the following statements:

"The image still does not clearly identify the fracture. It would have been better if the images were set to the optimal bone window configuration..."

"The windowing must be of concern as well. The exposure setting for the non-magnified view is different from the magnified ones. One must observe consistent windowing in order to assess the fractures more accurately."

"...the images which demonstrate a closer look on the otic capsule areas are not rendered in the temporal bone window which makes it difficult to assess."

"...aside from lack of standard windowing..." 


\section{PRACTICE PEARLS}

Although stated in very general terms, these statements all refer to the concept of WINDOWING, which is an important factor in computerized tomographic (CT) imaging. The window setting ('windowing') impacts on the ability to demonstrate the presence or absence of a lesion. The objective of this article is to present to the otolaryngologist some basic concepts underlying $\mathrm{CT}$ imaging. Particular emphasis will be placed on CT window and level settings and related concepts for non-radiologists in the interpretation and explanation of technical knowledge in the field of radiology. A reappraisal of the images in question based on the principles elaborated on will cap the discussion.

In 1895, Roentgen discovered x-rays and recognized its ability to variably penetrate or pass through objects, including parts of the human body, as captured on photographic plates and film. ${ }^{2}$ Since then, man has been able to visualize internal body structures in images rendered in different shades of black and white. How a structure appears on a radiograph depends on how much of the x-ray beam is absorbed or attenuated by the structure in question before it reaches the detector. Bones appear white because the calcium it contains absorbs or attenuates x-rays. Fat and other soft tissues absorb less and thus look gray. Air absorbs the least, so air filled structures like the lungs appear black (air filled normal mastoid air cells or paranasal sinuses appear grey, because these images actually pass through bony layers of the cranium). Although the distinction between air and bone are strikingly obvious, conventional x-rays cannot distinguish between soft tissues, because the more subtle variations between structures like the liver and pancreas are not clearly discernible. Since the radiograph records the mean absorption by all of the various structures that the $\mathrm{x}$-ray penetrates, quantitative measurements for individual soft tissue structures is not possible. ${ }^{3}$ This problem was surmounted by the development of computed tomography, a radiologic imaging modality that combines narrow beams of x-rays with computer technology in order to produce a detailed, cross-sectional image of an object of interest. A standard radiograph reflects an image obtained by passing a single, uni-directional x-ray beam through the body. On the other hand, computed tomography measures the attenuation of $x$-ray beams passing through sections of the body from a multitude of different angles. These measurements are processed by a computer in order to reconstruct an image of the body's interior and render it for viewing in greyscale on a monitor display. ${ }^{3}$

Structures are depicted on CT images as varying shades of gray, depending on the characteristic absorption or attenuation pattern that each tissue exhibits when exposed to ionizing radiation. The Hounsfield unit $(\mathrm{HU})$ is a relative quantitative scale of radio density which is used to display the range of tissue densities when viewing a CT scan. ${ }^{3,4}$
The scale is based on the density of water, which is arbitrarily defined to be zero Hounsfield units. It then ranges from the density of air, which is defined as $-1000 \mathrm{HU}$, to the density of bone at $+1000 \mathrm{HU}$. The denser the tissue, the more positive its value on the Hounsfield scale, and the brighter it appears on CT; the less dense the tissue, the more negative the value, and the darker it appears on the CT display. ${ }^{4}$

It can be seen from the scale of Hounsfield units for the most common soft tissues in the human body (Figure 2) that they occupy a very small portion of the entire scale, from around +20 to $+40 \mathrm{HU}$ for soft tissues and blood, to around -70 to $-90 \mathrm{HU}$ for fat. This represents a range of less than $150 \mathrm{HU}$.

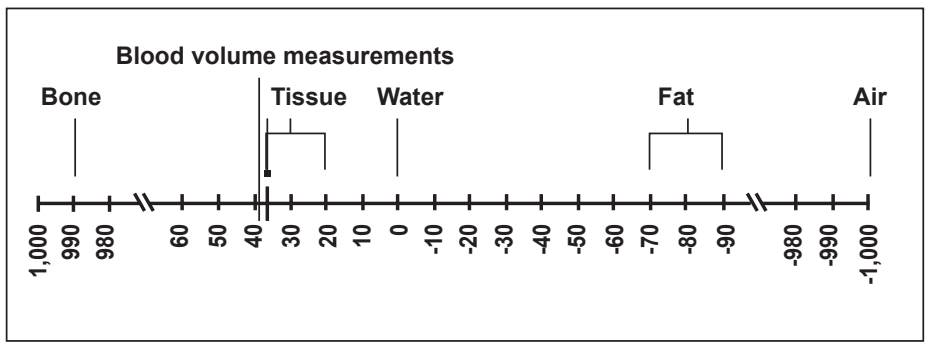

Figure 2. The Scale of Hounsfield units (HU) Adapted from Hounsfield $1980^{3}$

A standard monitor display like a cathode ray tube (CRT) or liquid crystal display (LCD) computer screen can display 256 shades of gray from black to white. Since the Hounsfield scale contains 2000 units from $+1000 \mathrm{HU}$ to $-1000 \mathrm{HU}$, each unit cannot be individually displayed on a monitor display. Each of the 256 separate shades of grey would have to represent a range of Hounsfield units, with a maximum of $7 \mathrm{HU}$ (approximate value of 2000 divided by 256) represented by a single shade of grey. Because of this, soft tissues whose representation in Hounsfield units are very close to each other would be nearly indistinguishable when the display depicts the entire range from air to bone. (Figure $3 A, B$ )
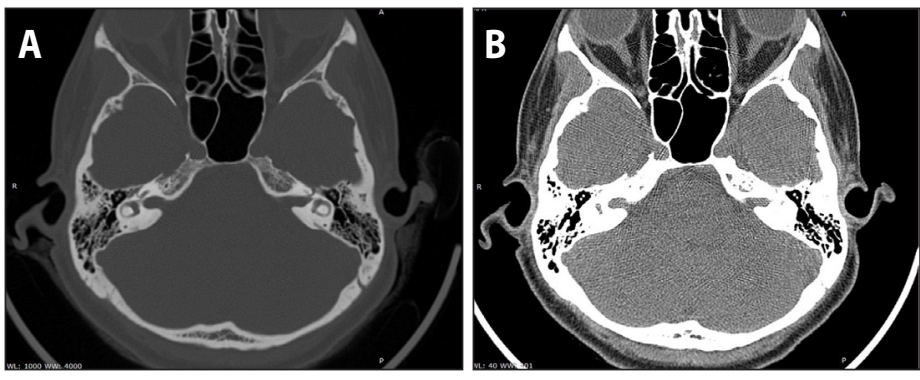

Figure 3. A. Axial CT image of the mastoids on a "bone window" setting that extends the range of $\mathrm{HU}$ values to 4000 . There is very little visually apparent difference in the appearance of the different soft tissue structures, including the brain, CSF, orbital muscles, fat and orbital contents; and B. Axial $\mathrm{CT}$ of the mastoids on a Soft Tissue Window of $400 \mathrm{HU}$. Note the clear delineation between the ocular muscles, periorbital fat, and intraocular soft tissues. The brain parenchyma and the CSF cannot be visually distinguished because it requires an even narrower window to achieve sufficient tissue contrast. 
In order to address this issue, the range of $\mathrm{HU}$ values depicted by the greyscale display can be manipulated to represent a much narrower range of numbers along the scale. This involves adjusting the window width (WW), which in standard CT terminology for image reconstruction and display is defined as the range of $\mathrm{CT}$ numbers (HU) that are distributed over the viewable grey scale of the display device or film. ${ }^{5}$ By doing so, each of the 256 shades of grey can represent a much smaller range of Hounsfield units, (Figure 4) and thus, increase the contrast between structures with very similar HU values (Figure 3 B). It does mean, however, that structures whose HU values lie outside of the range can only be depicted by being either very black or very white.

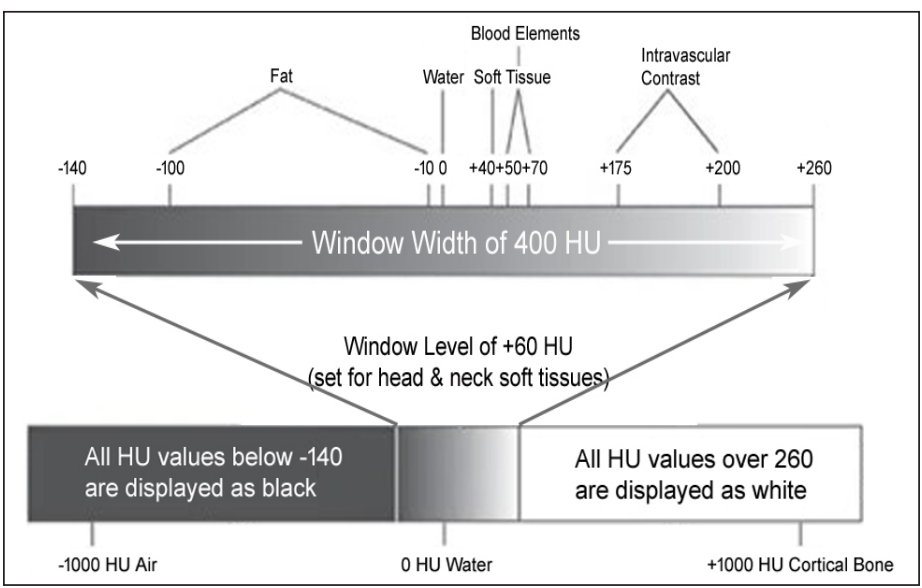

Figure 4. Concept of Window Width. In this case, a range of only $400 \mathrm{HU}$ is depicted by the grey scale display. Each of the individual shades of grey represent less than $2 \mathrm{HU}$, thus increasing the contrast between structures with very close $\mathrm{HU}$ values.

How does this relate to imaging structures within the temporal bone? It must be recognized that most of the important structures within the temporal bone are made of varying densities of bone surrounded by discrete pockets of air. Since these two are on opposite sides of the spectrum, a very wide window is necessary in order to depict them properly. In fact, the ideal window setting for the temporal bone was extended to represent $4000 \mathrm{HU}$, in order to accommodate the wide range of Hounsfield units that were subsequently discovered to represent the variations in bone density, which ranged from +700 for cancellous bone to $+3000 \mathrm{HU}$ for dense bone. ${ }^{6,7}$ An inappropriate window setting could render the fine structures within the temporal bone indistinguishable from each other. (Figure 5A, B)

Another key concept is the window level (WL) or window center. This is defined as the midpoint of the range of CT numbers (HU) displayed. ${ }^{8}$ Whatever lies at this level will appear in the middle of the greyscale, with those structures whose HU numbers lie below will
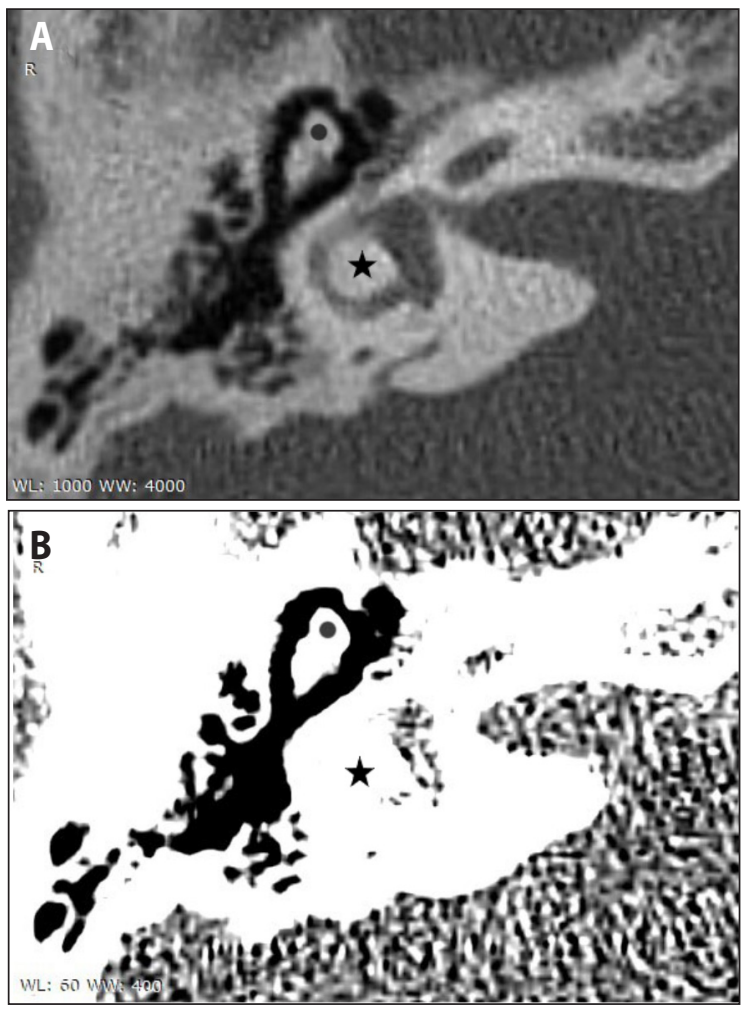

Figure 5. A. Axial CT of the temporal bone on a bone window setting (WW 4000 WL 1000). Note that the head of the malleus (black dot) can be clearly delineated from the adjacent incus. The central bony island of the lateral semicircular canal (black star) can be clearly delineated from the canal lumen, which has a gray appearance due to its fluid content; and B. Axial CT of the Temporal bone on a soft tissue window (WW 400, WL 60). Note that the head of the malleus (black dot) now appears as a homogenous white structure indistinct from the adjacent incus. The central bony island of the lateral semicircular canal (black star) and the surrounding canal lumen can no longer be distinguished from each other. *The WW and WL parameters are normally included in the information display of the DICOM study and is usually found in a corner. Depending on the imaging software, however, this information may be absent when viewing images in multiplanar reconstruction (MPR) mode.

appear on the darker side of the grey scale, while those whose HU numbers lie above will appear on the lighter side of the grey scale. Thus, the appearance of a structure can appear to be darker or lighter on the monitor display depending on the window level, despite their natural appearance in relation to the entire Hounsfield scale.

How do these concepts relate to the images in question? They relate to the obvious differences in the visual appearance of the two images. These differences will be highlighted in the following example, where the window settings of a totally different temporal bone imaging study set at approximately the same anatomic level were manipulated to simulate the appearance of the images in question.

Figure $6 \mathrm{~A}$ is an image set at the proper bone window setting for temporal bone studies, with a window width (WW) of 4000 and a window level (WL) or window center at 1000. The white arrow is pointing to a thin layer of bone that is clearly present overlying the 


\section{PRACTICE PEARLS}

round window niche. This image is similar to the larger, zoomed out view in the original images in question in Figure 1. It can be seen that, like the original image, the cochlear lumen appears grey, as it contains fluid. Figure $6 B$ appears to significantly transform the situation. By simply manipulating the window width (WW) to a much lower level close to 1000 , but maintaining the window level (WL) or window center at 1000 , the thin bone overlying the round window niche appears to have disappeared (white arrow), thus simulating the fracture supposedly identified in the magnified original image in Figure 1. How can we be certain that the two images have similar settings? Although the original image does not contain the window width and window level settings, two distinct features are obvious: the nearly homogenously white appearance of the bony structures, and the black appearance of the cochlear lumen. This appearance can be understood based on the
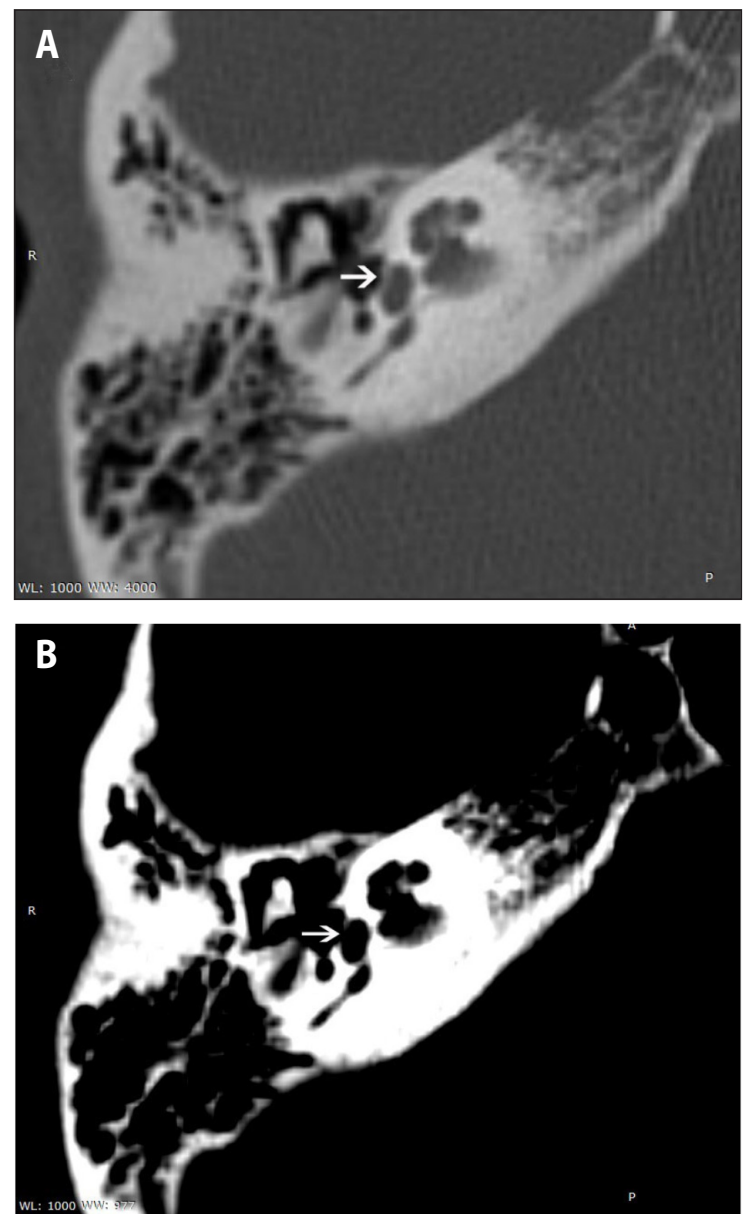

Figure 6. A. Axial CT of the temporal bone on a bone window setting (WW 4000 WL 1000) set at approximately the same anatomic level as the original images in question. The white arrow points to a thin layer of bone overlying the round window niche; and B. Axial CT of the temporal bone on an unconventional setting (WW near 1000, WL 1000). The white arrow points to the thin bone that appears to have disappeared, simulating the fracture identified in the original images in question. parameter changes. By decreasing the window width but maintaining the window level, a greater degree of contrast can be seen between thick bone and thin bone. As the midpoint did not change, the thin bone and the soft tissues within the cochlear lumen are now shifted to the lower spectrum of the grey scale and appear darker, thus explaining why the thin bone appears to have disappeared and the cochlear lumen appears black. This explains why a fracture can be misdiagnosed as being present when it actually does not exist.

In summary, this exercise in image evaluation brings out the importance of evaluating images using the correct window width and window level settings. Although computed tomographic imaging can allow visualization of even the tiniest bones in the human body with exquisite detail, these same details can be lost or misinterpreted by applying inappropriate imaging parameters. Parameters that can be so easily manipulated by a simple swipe of a mouse or the click of a button. Caveat utilitor!

1. Lacanilao RC, Chua RJ. Response from the authors. Philipp J Otolaryngol Head Neck Surg. 2020 May; 35(1):81. DOI: 10.32412/pjohns.v35i1.1261.

2. Underwood EA. Wilhelm Conrad Röntgen (1845-1923) and the Early Development of Radiology Proc R Soc Med. 1945 Oct; 38(12): 697-706. DOI: 10.1177/003591574503801214. PubMed PMID: 19993187, PubMed Central PMCID: PMC2181457.

3. Hounsfield GN. Computed medical imaging. Nobel lecture, December 8, 1979. J Comput Assist Tomogr. 1980 Oct; 4(5): 665-674. DOI: 10.1097/00004728-198010000-00017. PubMed PMID: 6997341.

4. Osborne T, Tang C, Sabarwal K, Prakash V. How to interpret an unenhanced CT Brain scan. Part 1: Basic principles of Computed Tomography and relevant neuroanatomy. South Sudan Med J. 2016 Aug; 9(3): 67-69.

5. American Association of Physicists in Medicine (AAPM) Working Group on Standardization of CT Nomenclature and Protocols. AAPM CT Lexicon version 1.3 04/20/2012. [cited 2020 Oct 29] Available from: https://www.aapm.org/pubs/CTProtocols/documents/CTTerminologyLexicon pdf.

6. Shaffer KA, Volz DJ and Haughton VM. Manipulation of CT Data for Temporal-Bone Imaging Radiology 1980 Dec; 137(3): 825-829. DOI: 10.1148/radiology.137.3.7444067. PubMed PMID: 7444067

7. Bibb R, Eggbeer D, Paterson A. Medical Modelling: The Application of Advanced Design and Rapid Prototyping Techniques in Medicine. 2nd Edition. Cambridge: Woodhead Publishing; 2015. DOI: 10.1016/B978-1-78242-300-3.00002-0.

8. Murphy A, et al. (co-authors not listed). Windowing (CT). Radiopaedia. [open-edit radiology resource on the Internet] Radiopaedia.org [cited 2020 Oct 30] Available from: https:// radiopaedia.org/articles/windowing-ct.
} 